\title{
Evaluation of the Relationships Between Computed Tomography Features, Pathological Findings, and Prognostic Risk Assessment in Gastrointestinal Stromal Tumors
}

\author{
Elsa Iannicelli, MD, * Francesco Carbonetti, MD, * Giulia Francesca Federici, MD, * Isabella Martini, MD, * \\ Salvatore Caterino, MD, † Emanuela Pilozzi, MD, $\neq$ Francesco Panzuto, MD, $\S$ Chiara Briani, MD, * \\ and Vincenzo David, $M D^{*}$
}

\begin{abstract}
Objectives: The aim of this study was to correlate computed tomography (CT) findings with pathology in gastrointestinal stromal tumors (GISTs). Methods: A retrospective evaluation of CT images of 44 patients with GISTs was performed. Computed tomography findings analyzed were location, size, margins, degree and pattern of contrast enhancement, angiogenesis, necrosis, signs of invasion, peritoneal effusion, peritoneal implants, surface ulceration, and calcifications.

Associations between CT features and mitotic rate, Miettinen classes of risk, lesions size, and among CT features were investigated. $\chi^{2}$ Test and Fisher test were performed.

Results: Mitotic rate was associated with margins $(P=0.016)$ and with adjacent organ invasion $(P=0.043)$. Pattern of contrast enhancement $(P=0.002)$, angiogenesis $(P=0.006)$, necrosis $(P=0.006)$, invasion of adjacent organs $(P=0.011)$, and margins $(P=0.006)$ were associated with classes of risk. Several associations $(P<0.05)$ between lesion size and CT features and among all the investigated $\mathrm{CT}$ features were found.

Conclusions: Computed tomography features could reflect GIST biology being associated with the mitotic rate and with classes of risk.
\end{abstract}

Key Words: digestive system neoplasm, gastrointestinal stromal tumors, multidetector computed tomography, risk assessment, mitotic index

(J Comput Assist Tomogr 2017;41: 271-278)

astrointestinal stromal tumors (GISTs) are rare tumors but the most common mesenchymal neoplasms of the gastrointestinal tract (GI). ${ }^{1,2}$

The term GIST was created to indicate a distinctive subgroup of gastrointestinal mesenchymal neoplasms with fusoid and epitheliod cells or rarely with pleomorphic cells of the GI; they are believed to originate from interstitial cells of Cajal or their stem cell-like precursors, and they usually express mutations of tyrosine kinase protein receptor (KIT) or platelet-derived growth factor receptor. ${ }^{2,3}$

The diagnosis of GIST is histological and implemented by the immunohistochemical marker CD117 (or C-KIT), which is positive in $95 \%$ of cases, whereas the other $5 \%$ of GISTs are positive for platelet-derived growth factor. ${ }^{4}$ These tumors have a large spectrum of biological behavior and are different from other solid lesions of the GI.

From the *Radiology Unit, $\uparrow$ Medical and Surgical Sciences and Translation Medicine, \$Pathology Department, and §Digestive and Liver Disease, Faculty of Medicine and Psychology, Sant'Andrea Hospital, Sapienza University of Rome, Rome, Italy.

Received for publication March 30, 2016; accepted May 23, 2016.

Correspondence to: Francesco Carbonetti, MD, Radiology Unit, Faculty of

Medicine and Psychology, Sant'Andrea Hospital, Sapienza University of

Rome, Via di Grottarossa 1035, Cap 00189, Rome, Italy

(e-mail: francescocarbonetti799@hotmail.com).

The authors declare no conflict of interest.

Copyright (C) 2016 Wolters Kluwer Health, Inc. All rights reserved.

DOI: $10.1097 /$ RCT.0000000000000499
Their prognosis is based on the Miettinen classification, recently reviewed, which takes into account location, size, and mitotic index of the tumor and stratifies the risk into 5 classes $^{4,5}$ (Fig. 1).

In this kind of tumor, the biological features play an important role for prognosis and evolution; imaging technique such as contrast-enhanced computed tomography (CECT) is a fundamental tool for detecting the lesion and staging and for the evaluation of the treatment response. ${ }^{6-11}$ Concerning the prognosis, CT can evaluate the tumor location and size.

The aim of our study was to investigate for any relations between the morphological GIST CT features and mitotic index, classes of risk, and lesion size and among all the evaluated CT parameters, to find whether any CT finding could give a first step orientation, before the pathological examination, of the biological behavior of the lesion.

Previous studies, where relations between CT features and pathological data were investigated, have been published, but none of them performed a comprehensive evaluation of the relations between CT features, mitotic index, classes of risk, and tumor size, as we propose in our article. ${ }^{10,12-15}$

\section{MATERIALS AND METHODS}

\section{Study Population}

Our local research ethics committee approved this study, and all patients provided written informed consent including information about the radiation exposure from the CT examinations.

This retrospective study was conducted reviewing the pathologic database of our hospital from January 2010 to November 2015 to select recorded cases with diagnosis of GIST. Seventynine patients were found.

Inclusion criteria for the study were the following: (1) pathologically confirmed diagnosis of GIST, (2) availability of a preoperative CECT, and (3) histological analysis of the surgical specimen reporting the lesion size, site of origin, and the mitotic index. Thirty-five patients were excluded from the analysis because CECT scan was not available in 30 patients and only biopsy was carried out in 5 patients. The final number of patients who matched the inclusion criteria and enrolled the study was 44 (26 men and 18 women; median age, 59 years; range, 30-88 years).

\section{Pathological Examination}

Histological diagnosis was based on microscopic morphology and immunophenotype. Immunohistochemistry was performed on freshly cut 3- $\mu \mathrm{m}$-thick, paraffin-embedded tissue sections using antibodies against C-KIT/CD117 (Dako A4502, polyclonal rabbit antihuman), according to manufacturer instructions. All investigated cases showed cytoplasmic/membranous positivity. Mitotic count was performed on 50 high power fields (HPFs) and expressed as number of mitoses per 50 HPFs. Neoplasm size was measured on formalin-fixed sample and was expressed in centimeters. 


\begin{tabular}{|l|l|l|l|l|l|}
\hline Size & Mitotic Rate & Gastric GISTs & Small intestinal GISTs & Duodenal GISTs & Rectal GISTs \\
\hline$\leq 2 \mathrm{~cm}$ & $\leq 5 / 50 \mathrm{HPFs}$ & None & None & None & None \\
\hline$>2 \leq 5 \mathrm{~cm}$ & $\leq 5 / 50 \mathrm{HPFs}$ & Very low & Low & Low & Low \\
\hline$>5 \leq 10 \mathrm{~cm}$ & $\leq 5 / 50 \mathrm{HPFs}$ & Low & Moderate & High & High \\
\hline$>10 \mathrm{~cm}$ & $\leq 5 / 50 \mathrm{HPFs}$ & Moderate & High & High & High \\
\hline$\leq 2 \mathrm{~cm}$ & $>5 / 50$ HPFs & Insufficient data & Insufficient data & Insufficient data & High \\
\hline$>2 \leq 5 \mathrm{~cm}$ & $>5 / 50 \mathrm{HPFs}$ & Moderate & High & High & High \\
\hline$>5 \leq 10 \mathrm{~cm}$ & $>5 / 50 \mathrm{HPFs}$ & High & High & High & High \\
\hline$>10 \mathrm{~cm}$ & $>5 / 50 \mathrm{HPFs}$ & High & High & High & High \\
\hline
\end{tabular}

FIGURE 1. Figure shows the GISTs' risk for progression based on tumor site, size, and mitotic rate according to Miettinen classification.

On the basis of mitotic count, neoplasm size, and site, all the patients were classified in 5 classes of risks (from none to high risk) and in an "insufficient data" group according to the Miettinen classification (Fig. 1). ${ }^{4,5}$

\section{CT Protocol}

Computed tomography scans were performed with multidetector CT equipment, GE Light Speed 16. The following technical parameters were used: $120 \mathrm{kV} ; 120$ to $180 \mathrm{~mA}$; gantry rotation time, 0.5 seconds; beam collimation, $16 \times 1.25 \mathrm{~mm}$; beam pitch, 1; reconstruction thickness, $2.5 \mathrm{~mm}$. The examinations were performed before and after intravenous administration of 120 to $150 \mathrm{~mL}$ of iodinated contrast medium with a rate speed of 3 to $3.5 \mathrm{~mL} / \mathrm{s}$. Arterial phase images were acquired 30 to 35 seconds after the injection, and portal venous phase was obtained with a delay of 70 to 80 seconds. Multiplanar reconstructions on coronal and sagittal planes and maximum intensity projection (MIP) were performed.

\section{Image Analysis}

The original and reconstructed CT images were reviewed on a picture archiving and communication system (PACS system, GE) by 2 radiologists in consensus reading, each with more than 10 years of experience in interpreting abdominal CT images, who recorded the following features: primary tumor location, lesion size, margins, growth pattern, intralesional necrosis, ulceration of the surface, degree and pattern of contrast enhancement, angiogenesis, invasion of adjacent organs, peritoneal implants, intralesional calcifications, and peritoneal effusion.

Lymphadenopathies were not evaluated because our study was not focused on the CT capability to detect metastatic lymph nodes. All the CT features were evaluated both on unenhanced and enhanced CT scans; the degree of contrast enhancement was analyzed and compared on unenhanced and portal phase images. Location was divided as follows: esophagus, stomach, duodenum, jejunum, ileum, colon, and mesentery. Tumor size, based on the maximal diameter on the CT axial plane, was classified into 4 classes according to the Miettinen classification: (1) less than or equal to $2 \mathrm{~cm}$, (2) greater than $2 \mathrm{~cm}$ but less than or equal to $5 \mathrm{~cm}$, (3) greater than $5 \mathrm{~cm}$ but less than or equal to $10 \mathrm{~cm}$, and (4) greater than $10 \mathrm{~cm}$. The margins of the lesions were defined as regular and irregular: the first one when the edge of the lesion appeared smooth, clear, and crisp and the latter when they looked jagged. Growth pattern was classified in endophytic, exophytic, or mixed considering lesion relations with the lumen of the digestive canal. Necrosis was assessed when intratumoral low-attenuation unenhanced areas were detected. Ulceration of the surface was referred when the endoluminal surface of the affected digestive tract showed irregular and discontinuous focal defect. The degree of contrast enhancement, which was measured as the change in attenuation on portal venous phase images relative to unenhanced images, was assessed.

For this purpose, 3 nonoverlapping regions of interest, 10 -mm sized, were drawn manually within solid tumor parts on unenhanced images and on venous phase images. The results were averaged for both measurements. The difference of density (delta of contrast enhancement, $\Delta$ ) between the mean HU values on enhanced and unenhanced CT images was obtained for each lesion. The statistic mean value of $\Delta$ was $55.33 \mathrm{HU}$; we considered as a mild to low enhanced lesion when the $\Delta$ was less than $55.33 \mathrm{HU}$, whereas high enhanced lesion was considered when a $\Delta$ greater than or equal to $55.33 \mathrm{HU}$ was observed.

Pattern of enhancement was assessed as homogenous or heterogeneous. Invasion of adjacent organs was evaluated: an illdefined boundary of the lesion, not clearly dissociated from surrounding structures, was considered as signs of invasion. Angiogenesis was assessed when enlarged and engorged blood vessels close to the lesion were detectable. Maximum intensity projection reconstruction was used to obtain better assessment or to evaluate the vascular anatomy. Peritoneal seeding was intended as a metastatic mass located in the peritoneum or in the mesentery and not close to the main tumor. The presence of intralesional calcifications and peritoneal effusion was also evaluated. A descriptive analysis of all CT parameters evaluated was carried out. The relations between $\mathrm{CT}$ features and mitotic rate, classes of risk, lesion size, and among all CT parameters were investigated.

\section{Statistical Analysis}

Numbers and percentage summarized categorical variables. Relationships between CT features and mitotic rate, CT features and classes of risk, CT features and lesion size, and among all CT parameters were tested by $\chi^{2}$ test or Fisher exact test, when needed. A $P<0.05$ was considered statistically significant. A professional statistician experienced with medical research performed all the analyses using STATA 13.0 (StataCorp LP, College Station, Tex).

\section{RESULTS}

Descriptive results of the CT features are reported in Table 1.

\section{Pathological Findings}

Among our cohort of 44 patients (pts), 11 pts (25\%) had lesions with mitotic index greater than 5 (with a maximum record of 
TABLE 1. Descriptive Results of the CT Features of GISTs

\begin{tabular}{|c|c|c|c|c|c|}
\hline & \multicolumn{2}{|c|}{ Pts } & & \multicolumn{2}{|c|}{ Pts } \\
\hline & $\mathbf{n}$ & $\%$ & & $\mathbf{n}$ & $\%$ \\
\hline Margins & & & Grow pattern & & \\
\hline Regular & 36 & $82 \%$ & Exophytic & 17 & $39 \%$ \\
\hline Irregular & 8 & $18 \%$ & Mixed & 13 & $30 \%$ \\
\hline Necrosis & & & Endophytic & 14 & $31 \%$ \\
\hline Present & 25 & $57 \%$ & C.E. pattern & & \\
\hline Absent & 19 & $43 \%$ & Homogeneous & 17 & $39 \%$ \\
\hline Surface ulceration & & & Heterogeneous & 27 & $61 \%$ \\
\hline Present & 13 & $30 \%$ & Location & & \\
\hline Absent & 31 & $70 \%$ & Stomach & 32 & $73 \%$ \\
\hline Adjacent organ invasion & & & Duodenum & 4 & $9 \%$ \\
\hline Present & 4 & $9 \%$ & Ileum & 3 & $7 \%$ \\
\hline Absent & 40 & $91 \%$ & Jejunum & 2 & $5 \%$ \\
\hline C.E. degree & & & Esophagus & 1 & $2 \%$ \\
\hline Mild-low: $\Delta H U<55.33$ & 22 & $50 \%$ & Mesentery & 1 & $2 \%$ \\
\hline High: $\Delta H U \geq 55.33$ & 22 & $50 \%$ & Colon & 1 & $2 \%$ \\
\hline Calcifications & & & Angiogenesis & & \\
\hline Present & 25 & $57 \%$ & Present & 14 & $32 \%$ \\
\hline Absent & 19 & $43 \%$ & Absent & 30 & $68 \%$ \\
\hline Peritoneal effusion & & & Size, cm & & \\
\hline Present & 5 & $11 \%$ & $\leq 2$ & 4 & $9 \%$ \\
\hline Absent & 39 & $89 \%$ & $>2$ but $\leq 5$ & 20 & $45 \%$ \\
\hline Peritoneal seeding & & & $>5$ but $\leq 10$ & 9 & $21 \%$ \\
\hline Present & 3 & $7 \%$ & $>10$ & 11 & $25 \%$ \\
\hline Absent & 41 & $93 \%$ & & & \\
\hline
\end{tabular}

C.E. indicates contrast enhancement; $\Delta \mathrm{HU}$, the statistic median value of the delta contrast enhancement.

mitoses of 180$)$, whereas the other 33 pts $(75 \%)$ had lesions with mitotic index less than or equal to 5 (lowest recorded number of mitoses, 0). Patients were divided, according to the reviewed Miettinen classification, into 5 classes of risk: 3 pts ( $8 \%$ ) as none risk, 12 pts (27\%) as very low risk, 9 pts (20\%) as low risk, 9 pts $(20 \%)$ as moderate risk, and 7 pts $(16 \%)$ as high risk. Four pts $(9 \%)$ of our sample who showed gastric, small bowel, or duodenal lesions sized $2 \mathrm{~cm}$ or smaller and with a mitotic index greater than $5 / 50 \mathrm{HPFs}$ were classified as insufficient data group as proposed by Miettinen and were not included in the statistical analysis between CT features and classes of risk (Fig. 1).

A total correspondence was found between $\mathrm{CT}$ findings and pathological data in the evaluation of the size of the lesions and location.

\section{Relations Between CT Features and Mitotic Rate}

The parameter "margins" was statistically associated with the mitotic index $(P=0.016)$ and with other organ invasion $(P=$ 0.043 ). Other not statistically significant correlations are fully detailed in Table 2.

\section{Relations Between CT Features and Risk Class}

Pattern of contrast enhancement $(P=0.002)$, angiogenesis $(P=0.006)$, necrosis $(P=0.006)$, adjacent organ invasion $(P=$ $0.011)$, and margins $(P=0.006)$ seemed to be statistically associated with the classes of risk. Our results demonstrated that the only CT parameter that showed a linear correlation with the increase of risk was the parameter "margins." Other not statistically significant correlations are fully reported in Table 3.

\section{Relations Between CT Features and Lesion Size}

Size of the lesion was associated with the pattern of contrast enhancement $(P=0.003)$, angiogenesis $(P=0.004)$, necrosis $(P=0.003)$, adjacent organ invasion $(P=0.009)$, and margins $(P<0.001)$. Other not statistically significant correlations are fully described in Table 4.

\section{Relations Among All the CT Features}

Several correlations were found among the CT features: pattern of contrast enhancement was associated with angiogenesis $(P=0.023)$, necrosis $(P<0.001)$, and margins $(P=0.013)$. Angiogenesis was associated with necrosis $(P=0.008)$, peritoneal implants $(P=0.027)$, and margins $(P=0.016)$. Necrosis, besides being associated with angiogenesis and pattern of contrast

TABLE 2. Relationships Between CT Features of GISTs and Mitotic Rate

\begin{tabular}{|c|c|c|c|c|c|}
\hline \multirow[b]{3}{*}{ CT Features } & \multicolumn{4}{|c|}{ Mitotic Rate } & \multirow[b]{3}{*}{$\boldsymbol{P}$} \\
\hline & \multicolumn{2}{|c|}{$\leq 5 / 50$ HPFs } & \multicolumn{2}{|c|}{$>5 / 50$ HPFs } & \\
\hline & $\mathbf{n}$ & $\%$ & $\mathbf{n}$ & $\%$ & \\
\hline & 33 & & 11 & & \\
\hline \multicolumn{6}{|c|}{ Enhancement pattern } \\
\hline Heterogeneous & 18 & 54.5 & 9 & 81.8 & 0.16 \\
\hline Homogeneous & 15 & 45.5 & 2 & 18.2 & \\
\hline \multicolumn{6}{|l|}{ Angiogenesis } \\
\hline Absent & 25 & 75.8 & 5 & 45.5 & 0.132 \\
\hline Present & 8 & 24.2 & 6 & 54.5 & \\
\hline \multicolumn{6}{|l|}{ Calcifications } \\
\hline Absent & 30 & 90.9 & 9 & 81.8 & 0.586 \\
\hline Present & 3 & 9.1 & 2 & 18.2 & \\
\hline \multicolumn{6}{|l|}{ Necrosis } \\
\hline Absent & 17 & 51.5 & 2 & 18.2 & 0.081 \\
\hline Present & 16 & 18.5 & 9 & 81.8 & \\
\hline \multicolumn{6}{|l|}{ Surface ulceration } \\
\hline Absent & 24 & 72.7 & 7 & 63.6 & 0.706 \\
\hline Present & 9 & 27.3 & 4 & 36.4 & \\
\hline \multicolumn{6}{|c|}{ Adjacent organ invasion } \\
\hline Absent & 32 & 97.0 & 8 & 72.7 & 0.043 \\
\hline Present & 1 & 3.0 & 3 & 27.3 & \\
\hline \multicolumn{6}{|l|}{ Peritoneal effusion } \\
\hline Absent & 31 & 93.9 & 8 & 72.7 & 0.051 \\
\hline Present & 2 & 6.1 & 3 & 27.3 & \\
\hline \multicolumn{6}{|l|}{ Peritoneal implants } \\
\hline Absent & 32 & 97.0 & 9 & 81.8 & 0.15 \\
\hline Present & 1 & 3.0 & 2 & 18.2 & \\
\hline \multicolumn{6}{|l|}{ Margins } \\
\hline Regular & 30 & 90.9 & 6 & 54.5 & 0.016 \\
\hline Irregular & 3 & 9.1 & 5 & 45.5 & \\
\hline \multicolumn{6}{|c|}{ Enhancement degree } \\
\hline Low-mild & 14 & 42.4 & 8 & 72.7 & 0.116 \\
\hline High & 19 & 57.6 & 3 & 27.3 & \\
\hline
\end{tabular}

In the column of the $P$ values, the statistical significant correlations are represented in bold. 
TABLE 3. Relationships Between CT Features of GISTs and Risk Classes

\begin{tabular}{|c|c|c|c|c|c|c|c|c|c|c|c|}
\hline \multirow[b]{4}{*}{ CT Features } & \multicolumn{10}{|c|}{ Risk Class } & \multirow[b]{4}{*}{$P$} \\
\hline & \multicolumn{2}{|c|}{$\mathbf{0}$} & \multicolumn{2}{|c|}{1} & \multicolumn{2}{|c|}{2} & \multicolumn{2}{|c|}{3} & \multicolumn{2}{|c|}{4} & \\
\hline & $\mathbf{n}$ & & $\mathbf{n}$ & & $\mathbf{n}$ & & $\mathbf{n}$ & & $\mathbf{n}$ & & \\
\hline & 3 & $\%$ & 12 & $\%$ & 9 & $\%$ & 9 & $\%$ & 7 & $\%$ & \\
\hline \multicolumn{12}{|c|}{ Enhancement pattern } \\
\hline Heterogeneous & 1 & 33.3 & 3 & 25.0 & 5 & 55.6 & 8 & 88.9 & 7 & 100.0 & \multirow[t]{2}{*}{0.002} \\
\hline Homogeneous & 2 & 66.7 & 9 & 75.0 & 4 & 44.4 & 1 & 11.1 & 0 & 0.0 & \\
\hline \multicolumn{12}{|l|}{ Angiogenesis } \\
\hline Absent & 3 & 100.0 & 10 & 83.3 & 8 & 88.9 & 5 & 55.6 & 1 & 14.3 & \multirow[t]{2}{*}{0.006} \\
\hline Present & 0 & 0.0 & 2 & 16.7 & 1 & 11.1 & 4 & 44.4 & 6 & 85.7 & \\
\hline \multicolumn{12}{|l|}{ Calcifications } \\
\hline Absent & 3 & 100.0 & 10 & 83.3 & 9 & 100.0 & 7 & 77.8 & 6 & 85.7 & \multirow[t]{2}{*}{0.706} \\
\hline Present & 0 & 0.0 & 2 & 16.7 & 0 & 0.0 & 2 & 22.2 & 1 & 14.3 & \\
\hline \multicolumn{12}{|l|}{ Necrosis } \\
\hline Absent & 2 & 66.7 & 9 & 75.0 & 5 & 55.6 & 2 & 22.2 & 0 & 0.0 & \multirow[t]{2}{*}{0.006} \\
\hline Present & 1 & 33.3 & 3 & 25.0 & 4 & 44.4 & 7 & 77.8 & 7 & 100.0 & \\
\hline \multicolumn{12}{|l|}{ Surface ulceration } \\
\hline Absent & 3 & 100.0 & 8 & 66.7 & 4 & 44.4 & 8 & 88.9 & 4 & 57.1 & \multirow[t]{2}{*}{0.211} \\
\hline Present & 0 & 0.0 & 4 & 33.3 & 5 & 55.6 & 1 & 11.1 & 3 & 42.9 & \\
\hline \multicolumn{12}{|c|}{ Adjacent organ invasion } \\
\hline Absent & 3 & 100.0 & 12 & 100.0 & 6 & 100.0 & 9 & 100.0 & 4 & 57.1 & \multirow[t]{2}{*}{0.011} \\
\hline Present & 0 & 0.0 & 0 & 0.0 & 3 & 33.3 & 0 & 0.0 & 3 & 42.9 & \\
\hline \multicolumn{12}{|l|}{ Peritoneal effusion } \\
\hline Absent & 3 & 100.0 & 12 & 100.0 & 8 & 88.9 & 7 & 77.8 & 6 & 85.7 & \multirow[t]{2}{*}{0.541} \\
\hline Present & 0 & 0.0 & 0 & 0.0 & 1 & 11.1 & 2 & 22.2 & 1 & 14.3 & \\
\hline \multicolumn{12}{|l|}{ Peritoneal implants } \\
\hline Absent & 3 & 100.0 & 12 & 100.0 & 9 & 100.0 & 8 & 88.9 & 6 & 85.7 & \multirow[t]{2}{*}{0.427} \\
\hline Present & 0 & 0.0 & 0 & 0.0 & 0 & 0.0 & 1 & 11.1 & 1 & 14.3 & \\
\hline \multicolumn{12}{|l|}{ Margins } \\
\hline Regular & 3 & 100.0 & 12 & 100.0 & 9 & 100.0 & 7 & 77.8 & 3 & 42.9 & \multirow[t]{2}{*}{0.006} \\
\hline Irregular & 0 & 0.0 & 0 & 0.0 & 0 & 0.0 & 2 & 22.2 & 4 & 57.1 & \\
\hline Enhancement degr & & & & & & & & & & & \\
\hline Low-mild & 1 & 33.3 & 5 & 41.7 & 3 & 33.3 & 7 & 77.8 & 4 & 57.1 & 0.332 \\
\hline High & 2 & 66.7 & 7 & 58.3 & 6 & 66.7 & 2 & 22.2 & 3 & 42.9 & \\
\hline
\end{tabular}

In the column of the $P$ values, the statistical significant correlations are represented in bold. This analysis was performed on 40 patients, belonging 4 patients to the "insufficient data group."

Risk classes: 0 , none risk; 1 , very low risk; 2 , low risk; 3 , moderate risk; 4 , high risk.

enhancement, was found to be statistically associated with margins $(P=0.007)$.

The parameter "margins," besides being associated with the parameter pattern of contrast enhancement, angiogenesis, and necrosis, was found to be statistically associated with invasion of adjacent organs $(P=0.001)$ and with peritoneal implants $(P=$ $0.004)$. The other not statistically significant correlations are fully described in Table 5 .

\section{DISUCSSION}

Gastrointestinal stromal tumors usually affect the stomach $(60 \%-70 \%)$ and small bowel $(20 \%-30 \%)$ and less frequently affect the esophagus $(5 \%)$, rectum, and colon $(5 \%)$; rarely, they could arise outside the GI (called e-GISTs). ${ }^{2-4}$ Their clinical presentation is extremely variable and depends on tumor location, biological features, and disease spread. Gastrointestinal stromal tumors might be asymptomatic, and diagnosis might be incidental during radiological, endoscopic, or surgical procedures. ${ }^{16-20}$ They usually arise from the intestinal wall, notably from the outer muscular layer, so they can have exophytic, endophytic, or mixed growth pattern with a size varying from several millimeters to greater than $30 \mathrm{~cm}$.

These tumors usually present areas of necrosis, cystic degeneration, or hemorrhage, phenomena often seen in larger lesions. ${ }^{6,7,10}$ Gastrointestinal stromal tumors have a large spectrum of behaviors, with a biologic potential at all sites of their occurrence. Miettinen and Lasota ${ }^{4}$ developed the currently used risk stratification. This classification combines tumor size, mitotic count, and tumor location and stratifies GIST prognosis into 5 classes (Fig. 1).

Few previous studies had investigated the correlation of GIST CT findings with pathology; however, to our knowledge, no similar studies focused on associations among CT features, mitotic rate, classes of risk, and tumor size had been carried out. ${ }^{10,12-15}$

Indeed, the latest article published on a similar topic by Pinaikul et al ${ }^{12}$ was mainly focused on the correlation between the CT features and the histological findings. 
TABLE 4. Relationships Between Size of GISTs and CT Features

\begin{tabular}{|c|c|c|c|c|c|c|c|c|c|}
\hline \multirow[b]{4}{*}{ CT Features } & \multicolumn{8}{|c|}{ Size } & \multirow[b]{4}{*}{$P$} \\
\hline & \multicolumn{2}{|c|}{$\leq 2 \mathrm{~cm}$} & \multicolumn{2}{|c|}{$>2 \mathrm{~cm}$ but $\leq 5 \mathrm{~cm}$} & \multicolumn{2}{|c|}{$>5 \mathrm{~cm}$ but $\leq 10 \mathrm{~cm}$} & \multicolumn{2}{|c|}{$>10 \mathrm{~cm}$} & \\
\hline & $\mathbf{n}$ & & $\mathbf{n}$ & & $\mathbf{n}$ & & $\mathbf{n}$ & & \\
\hline & 4 & $\%$ & 20 & $\%$ & 9 & $\%$ & 11 & $\%$ & \\
\hline \multicolumn{10}{|c|}{ Enhancement pattern } \\
\hline Heterogeneous & 1 & 25.0 & 8 & 40.0 & 7 & 77.8 & 11 & 100.0 & \multirow[t]{2}{*}{0.003} \\
\hline Homogeneous & 3 & 75.0 & 12 & 60.0 & 2 & 22.2 & 0 & 0.0 & \\
\hline \multicolumn{10}{|l|}{ Angiogenesis } \\
\hline Absent & 4 & 100.0 & 17 & 85.0 & 6 & 66.7 & 3 & 27.3 & \multirow[t]{2}{*}{0.004} \\
\hline Present & 0 & 0.0 & 3 & 15.0 & 3 & 33.3 & 8 & 72.7 & \\
\hline \multicolumn{10}{|l|}{ Calcifications } \\
\hline Absent & 4 & 100.0 & 17 & 85.0 & 9 & 100.0 & 9 & 81.8 & \multirow[t]{2}{*}{0.671} \\
\hline Present & 0 & 0.0 & 3 & 15.0 & 0 & 0.0 & 2 & 18.2 & \\
\hline \multicolumn{10}{|l|}{ Necrosis } \\
\hline Absent & 3 & 75.0 & 13 & 65.0 & 3 & 33.3 & 0 & 0.0 & \multirow[t]{2}{*}{0.003} \\
\hline Present & 1 & 25.0 & 7 & 35.0 & 6 & 66.7 & 11 & 100.0 & \\
\hline \multicolumn{10}{|l|}{ Surface ulceration } \\
\hline Absent & 4 & 100.0 & 13 & 65.0 & 5 & 55.6 & 9 & 81.8 & \multirow[t]{2}{*}{0.354} \\
\hline Present & 0 & 0.0 & 7 & 35.0 & 4 & 44.4 & 2 & 18.2 & \\
\hline \multicolumn{10}{|c|}{ Adjacent organ invasion } \\
\hline Absent & 4 & 100.0 & 20 & 100.0 & 9 & 100.0 & 7 & 63.6 & \multirow[t]{2}{*}{0.009} \\
\hline Present & 0 & 0.0 & 0 & 0.0 & 0 & 0.0 & 4 & 36.4 & \\
\hline \multicolumn{10}{|l|}{ Peritoneal effusion } \\
\hline Absent & 4 & 100.0 & 18 & 90.0 & 9 & 100.0 & 8 & 72.7 & \multirow[t]{2}{*}{0.262} \\
\hline Present & 0 & 0.0 & 2 & 10.0 & 0 & 0.0 & 3 & 27.3 & \\
\hline \multicolumn{10}{|l|}{ Peritoneal implants } \\
\hline Absent & 4 & 100.0 & 20 & 100.0 & 9 & 100.0 & 8 & 72.7 & \multirow[t]{2}{*}{0.048} \\
\hline Present & 0 & 0.0 & 0 & 0.0 & 0 & 0.0 & 3 & 27.3 & \\
\hline \multicolumn{10}{|l|}{ Margins } \\
\hline Regular & 4 & 100.0 & 20 & 100.0 & 9 & 100.0 & 3 & 27.3 & \multirow[t]{2}{*}{$<0.001$} \\
\hline Irregular & 0 & 0.0 & 0 & 0.0 & 0 & 0.0 & 8 & 72.7 & \\
\hline \multicolumn{10}{|c|}{ Enhancement degree } \\
\hline Low-mild & 1 & 25.0 & 9 & 45.0 & 5 & 55.6 & 7 & 63.6 & \multirow[t]{2}{*}{0.546} \\
\hline High & 3 & 75.0 & 11 & 55.0 & 4 & 44.4 & 4 & 36.4 & \\
\hline
\end{tabular}

In the column of the $P$ values, the statistical significant correlations are represented in bold.

TABLE 5. $P$ values of the Associations Among All the Investigated CT Features

\begin{tabular}{|c|c|c|c|c|c|c|c|c|c|}
\hline & Angiogenesis & Calcifications & Necrosis & $\begin{array}{c}\text { Surface } \\
\text { Ulceration }\end{array}$ & $\begin{array}{c}\text { Adjacent } \\
\text { Organ } \\
\text { Invasion }\end{array}$ & $\begin{array}{l}\text { Peritoneal } \\
\text { Effusion }\end{array}$ & $\begin{array}{l}\text { Peritoneal } \\
\text { Implants }\end{array}$ & Margins & $\begin{array}{c}\text { Enhancement } \\
\text { Degree }\end{array}$ \\
\hline Pattern of enhancement & 0.023 & 1.000 & $<0.001$ & 0.988 & 0.147 & 0.139 & 0.155 & 0.013 & 1.000 \\
\hline Angiogenesis & - & 0.647 & 0.008 & 0.923 & 0.581 & 0.677 & 0.027 & 0.016 & 1.000 \\
\hline Calcifications & 0.647 & - & 1.000 & 1.000 & 1.000 & 1.000 & 0.310 & 0.220 & 0.050 \\
\hline Necrosis & 0.008 & 1.000 & - & 0.797 & 0.122 & 0.060 & 0.247 & 0.007 & 1.000 \\
\hline Surface ulceration & 0.923 & 1.000 & 0.797 & - & 0.57 & 1.000 & 1.000 & 1.000 & 1.000 \\
\hline Adjacent organ invasion & 0.581 & 1.000 & 0.122 & 0.570 & - & 0.394 & 0.254 & 0.001 & 1.000 \\
\hline Peritoneal effusion & 0.677 & 1.000 & 0.060 & 1.000 & 0.394 & - & 0.310 & 0.219 & 1.000 \\
\hline Peritoneal implants & 0.027 & 0.310 & 0.247 & 1.000 & 0.254 & 0.310 & - & 0.004 & 0.233 \\
\hline Margins & 0.016 & 0.220 & 0.007 & 1.000 & 0.001 & 0.219 & 0.004 & - & 0.240 \\
\hline
\end{tabular}

The table shows the $P$ values, with the statistical significant ones in bold, obtained with the $\chi^{2}$ test by comparing the CT parameters with each other. 
Therefore, our work could be considered the first article where many features related to GIST prognosis and behavior are compared with CT findings to assess whether any CT findings could be predictive or specific of the Miettinen classes of risk. Therefore, we tried to give a useful contribution to our radiological daily practice. Results displayed in Table 2 show a statistically significant association between shape of lesion margins and mitotic index: most (90.9\%) of lesions with a number of mitosis less than or equal to $5 / 50 \mathrm{HPF}$ showed regular margins $(P=0.016)$, suggesting that solid lesions with smooth and not crispy borders could be less aggressive than the ones with jagged borders (Fig. 2).

All the tumors with irregular margins ( $8 \mathrm{pts}$ ) were sized greater than $10 \mathrm{~cm}$, and 5 of them showed high mitotic index (Fig. 3). To better analyze the relation between mitotic rate and margins, we calculated the mean number of mitoses in the group with a mitotic index greater than 5, taking into account lesions with both regular and irregular margins. We found out that the mean number of mitoses was higher (ie, 58.5) among the lesions with irregular margins compared with the mean value of mitoses (ie, 32.8) detected in neoplasms showing regular margins. This suggests that the mitotic index could be considered as a continuous variable as suggested by the monogram proposed by Rossi et al. ${ }^{20}$

Therefore, the relation between the mitotic index and the shape of the margins is influenced not only by the size of the lesion, as generally believed and confirmed by the analysis we had performed on lesions size and CT features (margins are associated with lesion size with a $P<0.001$ ), but also by the mitotic index.

The evidence that a pathological finding, such as the mitotic index, could also influence a CT detectable feature such the shape of the margins is quite interesting for future perspectives and further research.

This association was not proofed elsewhere neither in a previous recent study where the associations among the pathological findings and CT features were necrosis and peritoneal seeding. ${ }^{12}$ Thus, our result seems to be more interesting and quite new.

The mitotic index was also related $(P<0.043)$ with other organ invasion, but we do not consider this result original or impressive being the sample of lesions, which invaded other organs, was small (only 4 lesions) and quite obvious that the mitotic index could influence the tumor tendency to invade other organs.

It is worthwhile to point out that high contrast enhancement and intratumoral heterogeneity, which usually are CT findings suggestive of malignant lesions and reflect high cell density, hemorrhage, necrosis, and myxoid changes, in our study were not significantly related with the mitotic index. These results suggest that, in GISTs, these CT findings could be considered as independent features from the mitotic count although they resulted to be associated with the classes of risk and with the size of the lesions, which influence the biological behavior of GISTs as stated by Miettinen.

Several CT findings seemed to be significantly related with the risk of the aggressive clinical behavior of GISTs, such as pattern of contrast enhancement $(P=0.002)$, angiogenesis $(P=0.006)$, necrosis $(P=0.006)$, adjacent organ invasion $(P=0.011)$, and margins $(P=0.006)$ (Table 3, Fig. 3).

An interesting finding was that the presence of irregular margins showed a linear correlation with the risk classes, as it was absent in the none, very low, and low classes (Fig. 4), whereas
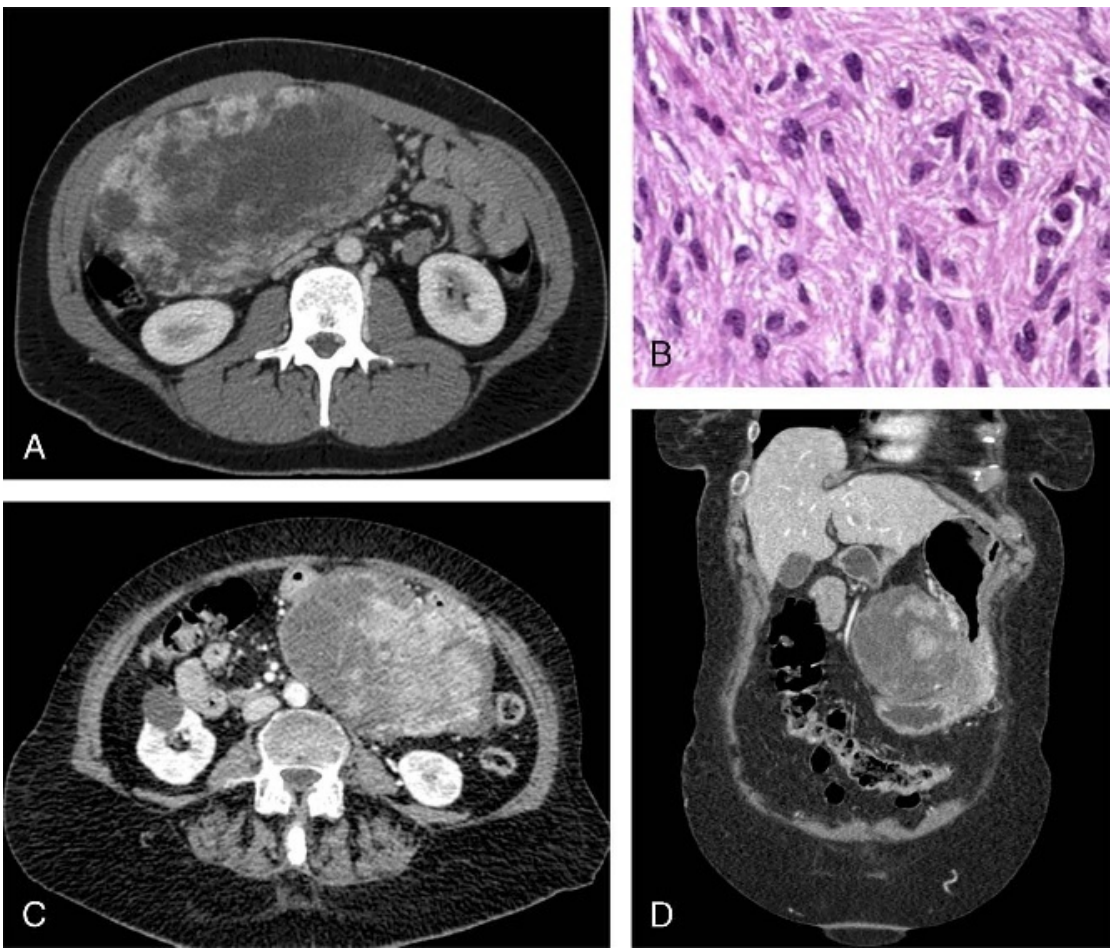

FIGURE 2. Gastric lesions both sized more than $10 \mathrm{~cm}$ with a low mitotic rate ( $\leq 5 / \mathrm{HPFs})$ of 2 different patients. A and B, A 39-year-old male patient with a gastric exophytic lesion, 17-cm sized, 2 mitoses/50 HPFs belonging to the moderate class of risk. A, Axial portal venous phase shows a lesion with regular margins, heterogeneous pattern of contrast enhancement, and wide necrosis inside. B, Photomicrograph (hematoxylin/eosin, $\times 400$ ) shows the low cellularity and very low mitotic rate. C and D, Similar CT features are depicted in another patient, a 84-year-old lady with gastric exophytic lesion, $13-\mathrm{cm}$ sized, 4 mitoses/50 HPFs belonging to the moderate class of risk. Portal venous phase in axial (C) and coronal (D) planes. Figure 2 can be viewed online in color at www.jcat.org. 

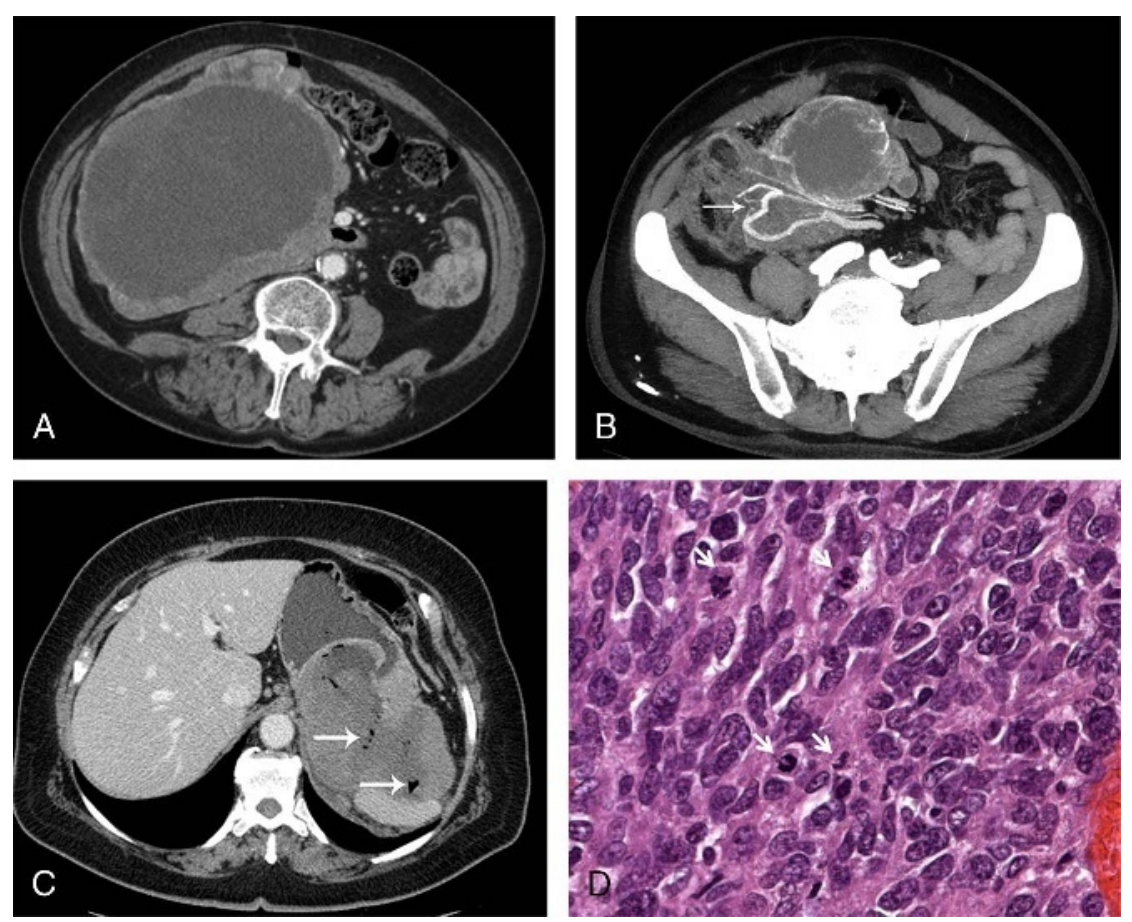

FIGURE 3. Gastric lesions both sized more than $10 \mathrm{~cm}$ with high mitotic rate ( $>5 / \mathrm{HPFs}$ ) of 2 different patients. A and B, A 68-year-old female patient with gastric exophytic lesion, $21-\mathrm{cm}$ sized, 40 mitoses $/ 50 \mathrm{HPFs}$, of the high risk class. A, Axial portal venous phase shows a lesion with irregular margins, heterogeneous pattern of contrast enhancement, and wide necrosis inside. B, MIP in axial arterial phase acquired in a lower level shows noticeable angiogenesis (arrow). C and D, A 70-year-old female patient with gastric lesion, 12-cm sized, 147 mitoses/50 HPFs, of the high class of risk. C, Axial portal venous phase shows a lesion with a mixed growth pattern, irregular margins, invasion of spleen, and heterogeneous pattern of contrast enhancement with necrotic areas inside (arrows). D, Photomicrograph (hematoxylin/eosin, $\times 400$ ) shows hypercellularity and very high mitotic rate (small single arrows). Figure 3 can be viewed online in color at www.jcat.org.

it could be observed in the $22.2 \%$ of the third class and in $57.1 \%$ of the fourth class.

This result strengthens the evidence that, with the increase of the risk, it increases the likelihood to detect irregular margins of the lesion on CT images.

The presence of heterogeneous pattern of contrast enhancement, angiogenesis, and necrosis was mainly observed in GISTs belonging to the moderate and high classes of risk, and they were respectively detected in $100 \%, 85.7 \%$, and $100 \%$ of the highest class. On the other hand, tumors belonging to the none and very low risk classes appeared in most cases as lesions with a homogenous pattern of contrast enhancement, without intratumoral necrotic areas or angiogenesis, and with regular margins. So, our study demonstrated that some imaging features could be considered as expression of a worst prognosis in GISTs.

Furthermore, this relation is supported by the significant associations of several CT features with the size of the tumor, which is 1 of the 3 main prognostic factors in Miettinen classification.

Tumor size seemed to be statistically significantly associated with the pattern of contrast enhancement, angiogenesis, necrosis, adjacent organ invasion, and margins (Table 4).
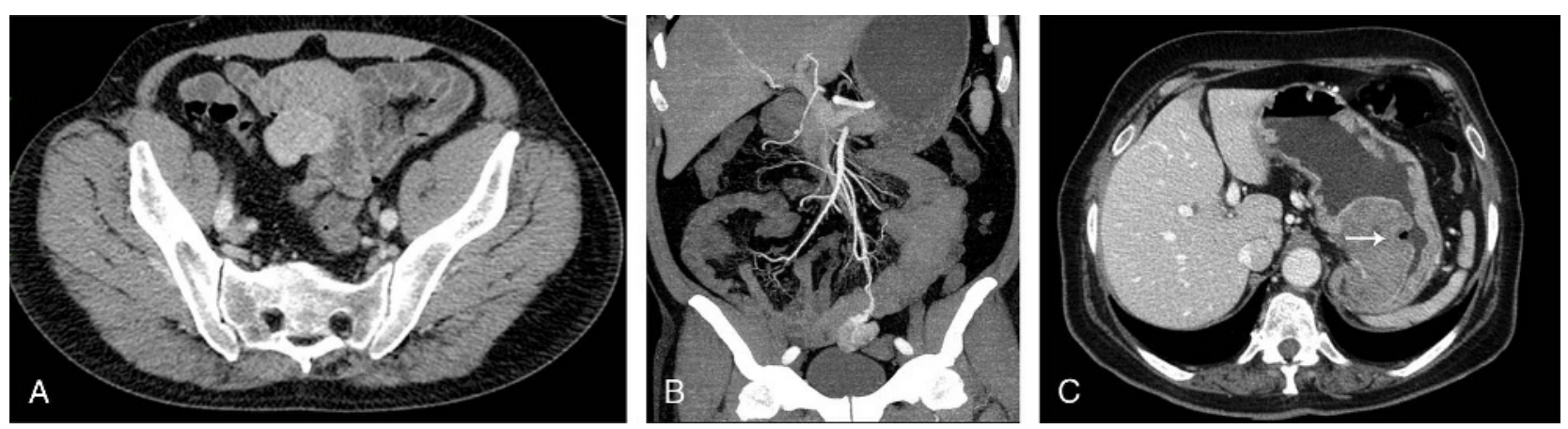

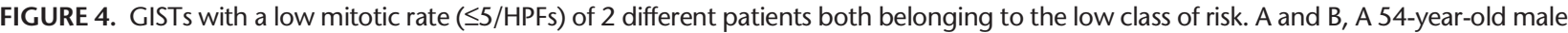
patient with exophytic ileal lesion, 4-cm sized, 2 mitoses/50 HPFs. A, Axial portal venous phase shows regular margins of the lesion and homogenous pattern of contrast enhancement. B, MIP in coronal arterial phase well depicts lesion's vascular supply by mesenteric superior artery. C, A 66-year-old female patient with gastric endophytic lesion, 7-cm sized, 5 mitoses/50 HPFs. Axial portal venous phase shows a lesion with regular margins, quite homogenous pattern of contrast enhancement, and surface ulceration (arrow). 
The analysis of the distribution of all these parameters among the different classes of size showed that heterogeneous contrast enhancement, irregular margins, and the other previously mentioned features trend to grow up with the increase of the size of tumor, being mostly detected in tumors sized 5 to 10 and greater than $10 \mathrm{~cm}$ (Fig. 3).

We only have to notice that the degree of contrast enhancement, usually considered, if high, as a remarkable characteristic of tumor biological activity, was not found to be associated with neither mitotic rate nor the class of risk, in accordance with previous articles. ${ }^{10,12-15}$ In addition, by analyzing the relation among all the CT features evaluated in this study (Table 5), a statistically significant association $(P<0.005)$ between pattern of contrast enhancement, necrosis, margins, and angiogenesis was found. It is interesting to notice that, in our sample, all tumors with irregular margins showed a heterogeneous enhancement and necrosis, and in $75 \%$ of them, angiogenesis was present.

We would stress the concept that the presence of irregular margins seems to be associated not only with the size of the lesion but also with the mitotic index, and it resulted to be the only CT feature that showed a linear correlation with the increase of the class of risk, making this relation of a strong and relevant importance.

If the aim of researches is to find possible CT features that really could reflect the clinical aggressiveness of GISTs, it is behoove to point out how much important is to correlate CT findings not only with the mitotic index, as done in previous studies, but also with size of the lesions and with the Miettinen classes of risk as proposed in our article.

Main limits of our study were as follows: (1) the study was designed as retrospective, and (2) the sample of patients was not equally distributed among the 2 mitotic index groups, thus resulting in 2 not homogenous classes: 11 pts with a high number of mitoses versus 33 pts with a low mitotic index. We could justify this limit considering that our article, to our knowledge, is the first attempt to research any relations between the imaging features of GISTs and its biological aggressiveness, expressed by mitotic rate and the prognostic risk assessment. Computed tomography is a fundamental tool in GIST evaluation and detection and contributes to the risk stratification evaluating the size of the tumor and their location; our article adds a value to the actual state of the art showing that CT morphological features could be relate not only with tumor size as yet known but also with pathological parameters, the mitotic rate, and the expression of the tumor biology. Even more interesting is the demonstration that several CT features are related with the risk assessment, suggesting that CT parameters could give a first step orientation, before the pathological examination, of the biological behavior of the lesion.

\section{ACKNOWLEDGMENTS}

The authors would like to thank Valentina Panetta, a professional statistician experienced with medical research, who performed all the statistical analyses.

\section{REFERENCES}

1. Rubin BP, Heinrich MC, Corless CL. Gastrointestinal stromal tumour. Lancet. 2007;369:1731-1741.
2. Al-Thani H, El-Menyar A, Rasul KI, et al. Clinical presentation, management and outcomes of gastrointestinal stromal tumors. Int J Surg 2014; $12: 1127-1133$

3. Min KW, Leabu M. Interstitial cells of Cajal (ICC) and gastrointestinal stromal tumor (GIST): facts, speculations, and myths. J Cell Mol Med. 2006;10:995-1013.

4. Miettinen M, Lasota J. Gastrointestinal stromal tumors: pathology and prognosis at different sites. Semin Diagn Pathol. 2006;23:70-83.

5. Miettinen M, Lasota J. Gastrointestinal stromal tumors. Gastroenterol Clin North Am. 2013;42:399-415.

6. Iannicelli E, Scavone G, Speranza A, et al. MDCT in GIST evaluation. Clin Ter. 2009;160:201-206.

7. Choi H. Response evaluation of gastrointestinal stromal tumors. Oncologist. 2008;13:4-7.

8. Aschoff AJ. MDCT of the abdomen. Eur Radiol. 2006;16:M54-M57.

9. Sandrasegaran K, Rajesh A, Rushing DA, et al. Gastrointestinal stromal tumors: CT and MRI findings. Eur Radiol. 2005;15:1407-1414.

10. Kim HC, Lee JM, Choi SH, et al. Imaging of gastrointestinal stromal tumours. J Comput Assist Tomogr. 2004;28:596-604.

11. Horton KM, Juluru K, Montogomery E, et al. Computed tomography imaging of gastrointestinal stromal tumours with pathology correlation. J Comput Assist Tomogr. 2004;28:811-817.

12. Pinaikul S, Woodtichartpreecha P, Kanngurn S, et al. 1189 Gastrointestinal stromal tumor (GIST): computed tomographic features and correlation of CT findings with histologic grade. J Med Assoc Thai. 2014;97:1189-1198.

13. Baheti AD, Shinagare AB, O'Neill AC, et al. MDCT and clinicopathological features of small bowel gastrointestinal stromal tumours in 102 patients: a single institute experience. Br J Radiol. 2015; 88:20150085.

14. Tateishi U, Hasegawa T, Satake M, et al. Gastrointestinal stromal tumor Correlation of computed tomography findings with tumor grade and mortality. J Comput Assist Tomogr. 2003;27:792-798.

15. Levy AD, Remotti HE, Thompson WM, et al. Gastrointestinal stromal tumors: radiologic features with pathologic correlation. Radiographics. 2003;23:283-304.

16. Boikos SA, Stratakis CA. The genetic landscape of gastrointestinal stromal tumor lacking KIT and PDGFRA mutations. Endocrine. 2014;47: 401-408.

17. Corless CL, Fletcher JA, Heinrich MC. Biology of gastrointestinal stromal tumors. J Clin Oncol. 2014;22:3813-3825.

18. Caterino S, Lorenzon L, Petrucciani N, et al. Gastrointestinal stromal tumors: correlation between symptoms at presentation, tumor location and prognostic factors in 47 consecutive patients. World J Surg Oncol. 2011;1: 9-13.

19. Choi YR, Kim SH, Kim SA, et al. Differentiation of large $(\geq 5 \mathrm{~cm})$ gastrointestinal stromal tumors from benign subepithelial tumors in the stomach: radiologists' performance using CT. Eur J Radiol. 2014;83: 250-260.

20. Rossi S, Miceli R, Messerini L, et al. Natural history of imatinib-naive GISTs: a retrospective analysis of 929 cases with long-term follow-up and development of a survival nomogram based on mitotic index and size as continuous variables. Am J Surg Pathol. 2011;35:1646-1656. 